\title{
Computational neuroanatomy and co-expression of genes in the adult mouse brain, analysis tools for the Allen Brain Atlas
}

\author{
Pascal Grange ${ }^{1, *}$, Michael Hawrylycz ${ }^{2}$ and Partha P. Mitra ${ }^{1}$ \\ ${ }^{1}$ Cold Spring Harbor Laboratory, Cold Spring Harbor, New York, NY 11724, USA \\ 2 Allen Institute for Brain Science, Washington, WA 98103, USA \\ * Correspondence: pascal.grange@polytechnique.org
}

Received November 16, 2012; Accepted January 2, 2013

\begin{abstract}
We review quantitative methods and software developed to analyze genome-scale, brain-wide spatiallymapped gene-expression data. We expose new methods based on the underlying high-dimensional geometry of voxel space and gene space, and on simulations of the distribution of co-expression networks of a given size. We apply them to the Allen Atlas of the adult mouse brain, and to the coexpression network of a set of genes related to nicotine addiction retrieved from the NicSNP database. The computational methods are implemented in BrainGeneExpressionAnalysis (BGEA), a Matlab toolbox available for download.
\end{abstract}

\section{INTRODUCTION AND BACKGROUND}

The mammalian brain is a structure of daunting complexity, whose study started millenia ago and has been recently renewed by molecular biology and computational imaging [1]. The Allen Brain Atlas (ABA), the first Web-based, genome-wide atlas of gene expression in the adult mouse brain, was a large-scale experimental effort [2-7]. The resulting data set consists of co-registered in situ hybridization (ISH) image series for thousands of genes. It is now available to neuroscientists worldwide, and has given rise to the development of quantitative techniques and software for data analysis. The present paper reviews recent developments that have been applied to co-expression studies in the mouse brain and are publicly available for use on the Web [8] and on the desktop [9].

On the other hand, lists of condition-related genes are now available from databases that pool results of different studies [10,11]. As these studies employ different methods and result in lists of hundreds of genes, it is important to investigate any possible order (or lack of it) in these lists. The Allen Brain Atlas provides ways to do this, by studying brain-wide co-expression of genes, and by enabling to compare gene expression to classical neuroanatomy, in a genome-scale data set based on a unified protocol.

Advanced data exploration tools have already been developed for the Allen Brain Atlas. NeuroBlast allows users to explore the correlation structure between genes in the ABA. It was inspired by the Basic Local Alignment Research Tool [12], which derives lists of similar genes to a given gene at the level of sequences, and transposed the technique to the analysis of similarity between patterns of gene expression in the brain [13]. The Anatomic Gene Expression Atlas (AGEA) [14] was launched in 2007. It is based on the spatial correlation of the atlas. The user can explore three-dimensional correlation maps based on correlations between voxels, computed using thousands of genes, and retrieve hierarchical data-driven parcellations of the brain.

The Weighted Gene Co-Expression Network Analysis framework (WGCNA) has been used to isolate clusters of genes from correlations between multiple microarray samples. In this approach the gene networks are typically constructed from the correlation coefficients of microarray data, from which graphs are constructed and thresholded at a value chosen as to satisfy certain statistical criteria $[15,16]$. However, in the case of the Allen Brain Atlas, gene-expression data are scaffolded by 
classical neuroanatomy, since ISH data are co-registered to the Allen Reference Atlas (ARA) [17]. The whole brain is voxelized, and the voxels are annotated according to the brain region to which they belong, which allows to compare the expression of sets of genes to brain regions (see Figure 1 and Ref. [18]). Hence we developed computational methods to:

1. study the whole range of co-expression values between pairs of genes;

2. use the Allen Atlas as a probabilistic universe to estimate the distribution of co-expression networks;

3. compare the expression patterns of highly coexpressed sets of genes to classical neuroanatomy.

These methods are implemented in BrainGeneExpressionAnalysis (BGEA), a Matlab toolbox downloadable from www.brainarchitecture.org. They are applied to a set of 288 genes extracted from the NicSNP database, which have been linked to nicotine dependence, based on the statistical significance of allele frequency difference between cases and controls, and for which mouse orthologs are found in the coronal Allen Atlas of the adult mouse brain.

\section{METHODS}

The spatial frequency of tissue-sectioning in the experimental pipeline of the Allen Brain Atlas corresponds to slices with a thickness of $100 \mu \mathrm{m}$. Each section was registered to a grid with a resolution of 100 microns $[19,20]$. The induced three-dimensional grid was subsampled to a resolution of 200 microns in order to increase the overlap between different experiments. This procedure results in a partition of the mouse brain into $V=$ 49742 cubic voxels. We focus on the co-registered quantities obtained at a spatial resolution of $200 \mu \mathrm{m}$, for several thousands of genes, after subsampling. In particular, the expression energy of each gene labeled $g$ in the Atlas was defined and computed [14] at each voxel labeled $v$ in the mouse brain:

$$
E(v, g)=\frac{\Sigma_{p \in v} M(p) I(p)}{\Sigma_{p \in v} 1},
$$

where $p$ is a pixel index, and the denominator counts the pixels that are contained in the voxel $v$ for the ISH image series of gene $g$. The quantity $M(p)$ is a Boolean segmentation mask that takes value 1 at pixels classified as expressing the gene, and 0 at other pixels. The quantity $I(p)$ is the grayscale value of the pixels in ISH images. The present paper uses the voxel-by-gene matrix of expression energies $E$ as the digitized version of the Allen Brain Atlas. The expression energies of the genes in the full coronal and sagittal atlas can be downloaded using the Web service provided by the Allen Institute [21].

\section{Brain-wide co-expression networks: graph properties}

The statistical study of brain-wide co-expression networks using BGEA is summarized in the flowchart of Figure 2. Detailed examples of the use of the software are provided in the toolbox manual [9]. The columns of the matrix $E$ of expression energies of Eq. (1) are naturally identified to vectors in a $V$-dimensional space (the voxel space). Given two genes, the two corresponding columns of the matrix $E$ span a two-dimensional vector space. The simplest geometric quantity to study for this system is the angle between the two vectors. As all the entries of the matrix $E$ are positive by construction, this angle is

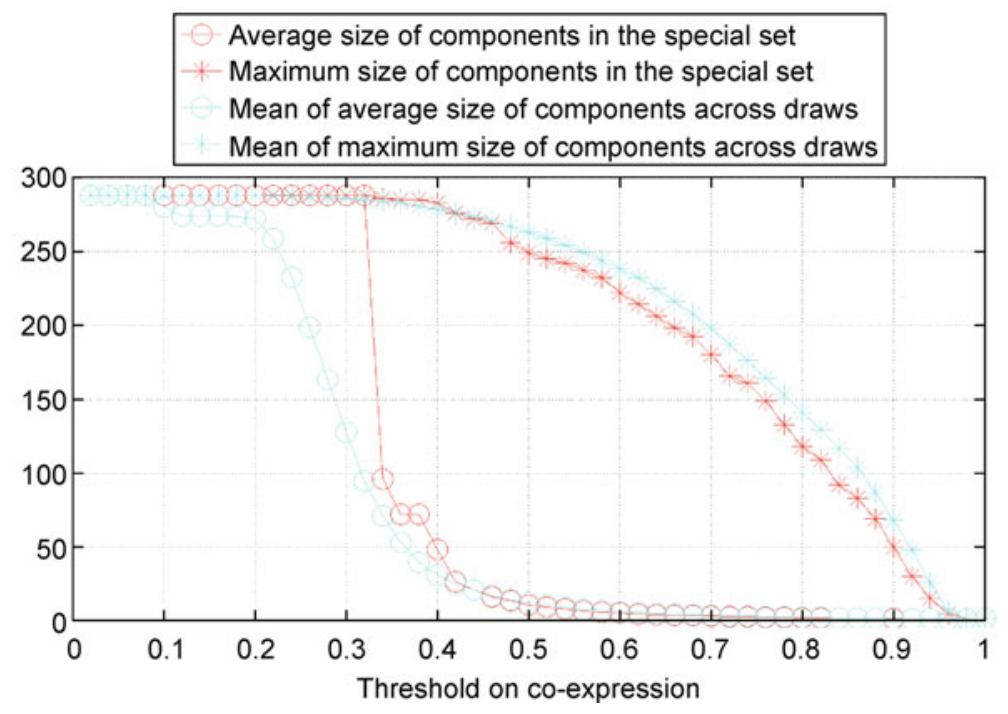

Figure 1. Monte Carlo analysis of the graph underlying the co-expression matrix of 288 genes from the NicSNP database. Average and maximum size of connected components as a function of the threshold on co-expression. 
between 0 and $\pi / 2$. The angle between the two vectors is therefore completely characterized by its cosine, which is readily expressed in terms of expression energies. This cosine similarity, defined in Eq. (2), for genes labeled $g$ and $g^{\prime}$, is called the co-expression of genes $g$ and $g^{\prime}$.

$$
\operatorname{coExpr}\left(g, g^{\prime}\right)=\sum_{v=1}^{V} \frac{E(v, g) E\left(v, g^{\prime}\right)}{\sqrt{\Sigma_{u=1}^{V} E(u, g)^{2} \Sigma_{w=1}^{V} E(w, g)^{2}}} .
$$

The more co-expressed $g$ and $g^{\prime}$ are in the brain, the closer their cosine similarity is to 1 .

Once the co-expressions have been computed for all pairs of genes in the Allen Brain Atlas, they are naturally arranged in a matrix, denoted by $C^{\text {Atlas }}$, with the genes arranged in the same order as the list of genes in the atlas:

$$
C^{\text {Atlas }}\left(g, g^{\prime}\right)=\operatorname{coExpr}\left(g, g^{\prime}\right), \quad 1 \leqslant g, g^{\prime} \leqslant G_{\text {Atlas }},
$$

where $G_{\text {Atlas }}$ is the total number of genes included in the data set (see the Applications section for more details on this choice). The matrix $C^{\text {Atlas }}$ is symmetric and its diagonal entries are all equal to 1 . This diagonal is trivial in the sense that it expresses the perfect alignment of any vector in voxel space with itself. When we consider the distribution of the entries of the co-expression matrix, we really mean the distribution of the upper-diagonal coefficients.

Given a set of genes (with $G_{\text {set }}$ elements) curated from the literature, possibly coming from different studies, one may ask if the brain-wide expression profiles of these genes (or a subset thereof) are closer to each other than

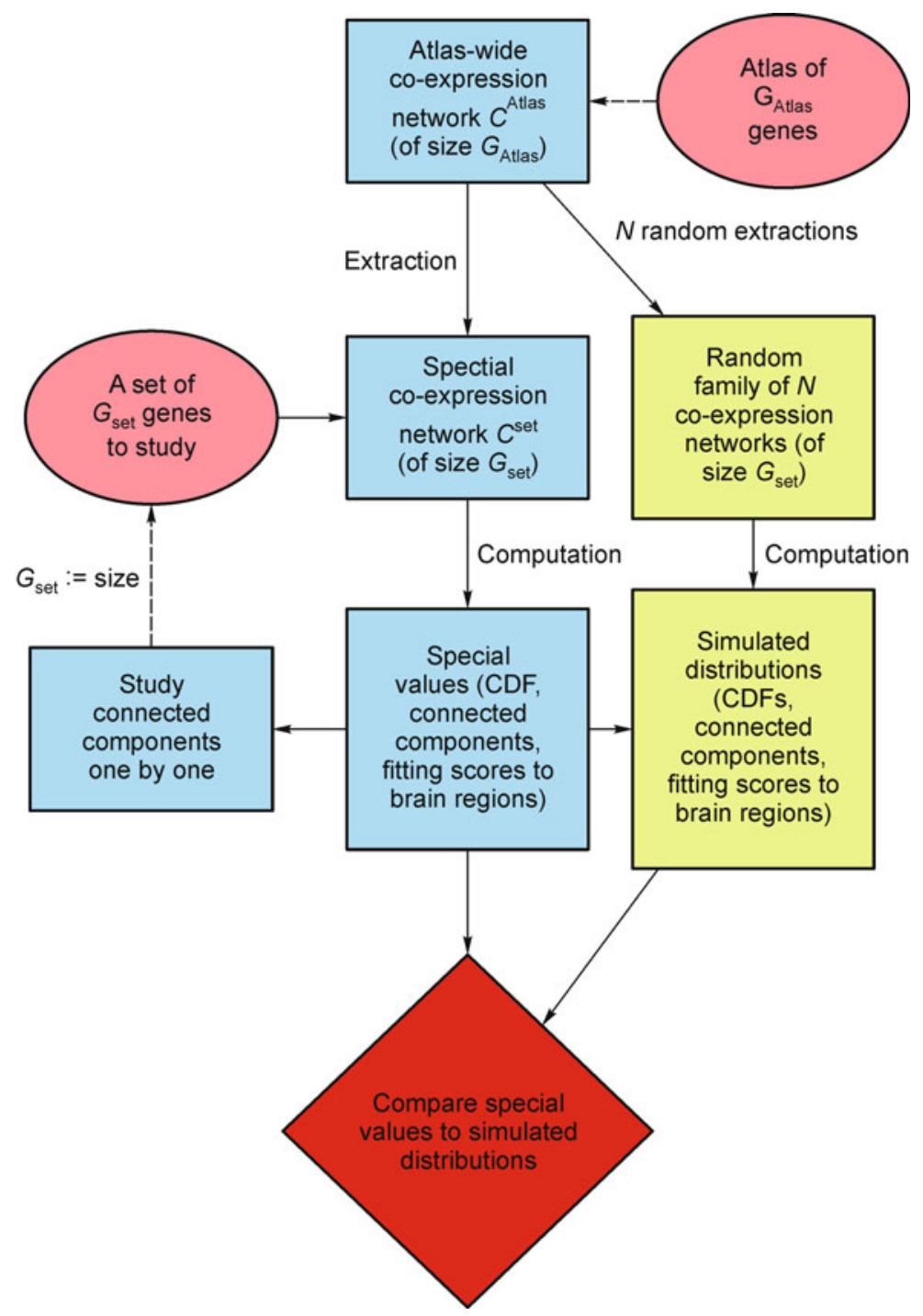

Figure 2. The flowchart of computational analysis of the collective neuroanatomical properties of a set of genes in the BrainGeneExpressionAnalysis toolbox. The steps marked as random extractions and computations are described in supplementary materials S2 and S3. 
expected by chance, using the full atlas as a probabilistic universe. The set of genes for which brain-wide expression data are available from the Allen Atlas of the adult mouse brain consists of 4104 genes, which is of the same order of magnitude as the total number of genes in the mouse genome. The number of sets of genes of a given size that can be drawn from the atlas therefore grows quickly with the size of the set. To study the coexpression properties of the chosen set of genes, a $G_{\text {set }}{ }^{-}$ by- $G_{\text {set }}$ matrix $C^{\text {set }}$ can be extracted from the whole coexpression matrix $C^{\text {Atlas }}$. A set of strongly co-expressed genes corresponds to a matrix $C^{\text {set }}$ with large coefficients.

To formalise this idea, we propose to study the matrix in terms of the underlying graph. There are $G_{\text {Atlas }}$ ways of ordering the genes in the Atlas. They give rise to different co-expression matrices, related by similarity transformation. But the sets of highly co-expressed genes are invariant under these transformations. The co-expression matrix can be mapped to a weighted graph in a straightforward way. The vertices of the graph are the genes, and the edges are as follows:

- Genes $g$ and $g^{\prime}$ are linked by an edge if their coexpression $\operatorname{coExpr}\left(g, g^{\prime}\right)$ is strictly positive.

- If an edge exists, it has weight $\operatorname{coExpr}\left(g, g^{\prime}\right)$.

We have to define large co-expression matrices in relative terms, using thresholds on the value of coexpression that describe the whole set of possible values. The entries of the co-expression matrices are numbers between 0 and 1 by construction. We define the following thresholding procedure on co-expression graphs: Given a threshold $\rho$ between 0 and 1 , and a co-expression matrix (which can come from any set of genes in the Allen Atlas), put to zero all the coefficients that are lower than the threshold (see Figure 3 for an illustration on a toymodel with 9 genes).

The graph corresponding to a co-expression matrix has connected components, and each connected component has a certain number of genes in it. The graph properties of the matrix $C^{\text {set }}$ can be studied by computing the average and maximal size of the connected components at every value of the threshold using Tarjan's algorithm [22]. This induces functions of the threshold that can be compared to those obtained from $N$ random sets of genes of the same size $G_{\text {set }}$ (these computations on random sets of genes correspond to the two rectangular boxes on the right of the flowchart in Figure 2, see Supplementary Materials S2 for mathematical details).

\section{Cumulative distribution functions of co-expression}

To complement the graph-theoretic approach, we can study the cumulative distribution function of the entries of the co-expression matrix of the set of genes, and compare it to the one resulting from random sets of genes of the same size (see Supplementary Materials S2 and S3 for mathematical details). For every number between 0 and 1 , the empirical cumulative distribution function of $C^{\text {set }}$, denoted by $\mathrm{CDF}^{\text {set }}$ is defined as the fraction of the entries
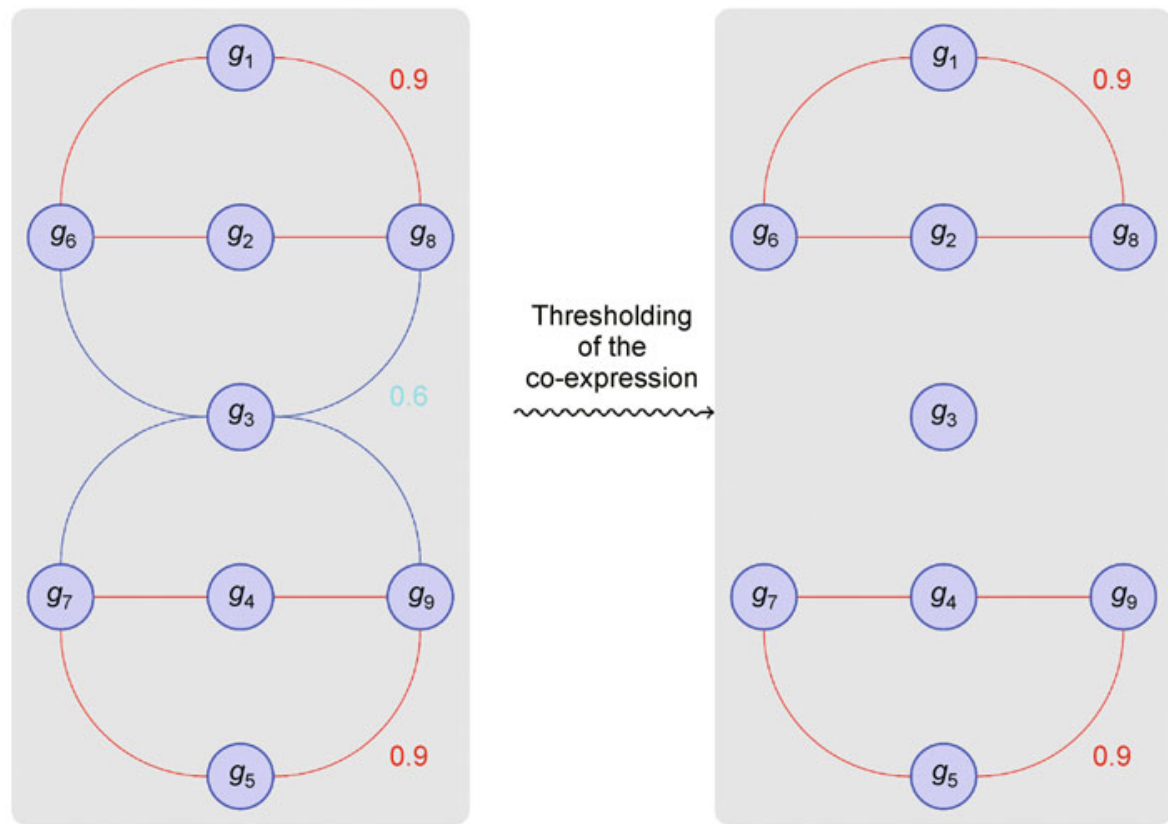

Figure 3. A toy model with 9 genes, and only three distinct values of co-expression, $0,0.6$ and 0.9 , for simplicity. Before any thresholding procedure is applied (on the left-hand side of the figure), there is one connected component. The average and maximum size of connected components are both 9 . The graph on the right-hand side is obtained by a thresholding procedure at a threshold of 0.6. There are three connected components, the maximal size is 4 , and the average size is 3 . 
of the upper-diagonal part of the co-expression matrix that are smaller than this number.

To compare the co-expression network of interest $C^{\text {set }}$ to random networks of the same size, the procedure is exactly the same as with the thresholded matrices, except that the quantities computed from the $N$ random draws are cumulative distribution functions rather than connected components (see Supplementary Materials for mathematical details). For each random set of $G_{\text {set }}$ genes drawn from the Allen Atlas, one can compute the empirical distribution function of the corresponding submatrix of $C^{\text {Atlas }}$, and average over the draws. The average over the draws converges toward the one of a typical network of $G_{\text {set }}$ genes when the number of random draws is sufficiently large.

\section{Comparison to classical neuroanatomy}

Given a brain region $\omega_{r}, 1 \leqslant r \leqslant R$, where $R$ is the number of brain regions in the Allen Reference Atlas [17] (to which gene expression data are registered), the fitting score of a brain-wide function $f$ in this region, or $\phi_{r}(f)$ can be defined [18] as the cosine distance between this function and the characteristic function of the region. It is formally the same as the co-expression of a gene whose expression energy would be the brain-wide function, and another gene that would be uniformly expressed in the region, and nowhere else:

$$
\phi_{r}(f)=\sum_{v \in \Omega} \frac{f(v) 1\left(v \in \omega_{r}\right)}{\sqrt{\Sigma_{u \in \Omega} f(u)^{2}} \sqrt{\Sigma_{w \in \Omega} 1\left(w \in \omega_{r}\right)^{2}}} .
$$

The distribution of fitting scores in all the brain regions for sets of $G_{\text {set }}$ genes can be simulated by the Monte Carlo methods described in Supplementary Materials S4.

Even though clustering methods [23] have shown that the correspondence between large sets of genes and brain regions in the Allen Atlas is not perfect, it is possible to detect small subsets of a set of genes curated from the literature that have exceptionally good fitting properties in some brain regions (see Figure 4 for an example of a set of 3 genes detected to fit the striatum significantly better than expected by chance).

\section{APPLICATIONS}

\section{Choice of genes: coronal and sagittal atlases}

The notion of an atlas of gene expression in the adult mouse brain rests on the assumption that there is a constant component across all brains at the final stage of development (the developmental atlas addresses the challenge of measurement of this component at earlier stages [24]). For an account of the standardization process that began in 2001 and led to the data generation and release of the Allen Brain Atlas, see Ref. [6].

The issue of reproducibility of ISH data can be addressed in several ways during the analysis of data. In NeuroBlast, the user can specify a given image series as input. The BrainGeneExpressionAnalysis toolbox is based on the analysis of the matrix of expression energies defined in Eq. (1), whose columns consist of brain-wide gene-expression data. This restricts the choice of genes to be analyzed in by BGEA to the 4104 genes for which a brain-wide, coronal atlas was developed. For these genes, sagittal, registered data are also available in the left hemisphere. We computed correlation coefficients between sagittal and coronal data. The correlation coefficients are not all positive. Sagittal data sets usually come from brain sections taken from the left hemisphere only. Hence the computation of correlation between (coregistered) sagittal and coronal data has to be restricted to the voxels belonging to the left hemisphere. For each gene $g$ in the coronal atlas, we computed the following correlation coefficient between sagittal and coronal data:

$$
\rho_{\text {sagittal } / \text { coronal }}(g)=\frac{\Sigma_{v \in \text { left hemisphere }} E_{\text {sagittal }}(v, g) E_{\text {coronal }}(v, g)}{\sqrt{\Sigma_{v \in \text { left hemisphere }} E_{\text {sagittal }}(v, g)^{2} \Sigma_{v \in \text { left hemisphere }} E_{\text {coronal }}(v, g)^{2}}},
$$

where $E_{\text {sagittal }}$ and $E_{\text {coronal }}$ are the voxel-by-gene matrices of Eq. (1) for sagittal and coronal data respectively. The results are shown on Figure 5. Some genes have negative correlation between sagittal and coronal data. The gene with highest value of $\rho_{\text {sagittal/coronal }}$ is Tcf712. The present study focuses on genes for which the correlation is larger than the 25 th percentile of the distribution of $\rho_{\text {sagittal/coronal }}$.

This set of $G_{\text {Atlas }}:=3041$ genes serves as a reference set to which special sets of genes can be compared using the methods described above. In particular, this choice excludes all the genes with negative correlation. Other user-defined choices of genes are possible within the coronal atlas. They can be implemented by modifying the data matrix defined in Eq. (1) and the list of genes corresponding to its columns in BGEA.

The sorted entries of the upper-diagonal part of the induced co-expression matrix $C^{\mathrm{Atlas}}$ are plotted on Figure 6A. The pair of genes with highest co-expression are Atp6v0c and Atp2a2, whose expression profiles are plotted on Figures $6 \mathrm{~B}$ and $6 \mathrm{C}$. The profile of coexpressions is fairly linear, except at the end of the spectrum, which motivates a uniform exploration of the interval $[0,1]$ when studying co-expression networks (see the pseudocode in Supplementary Materials S2). 


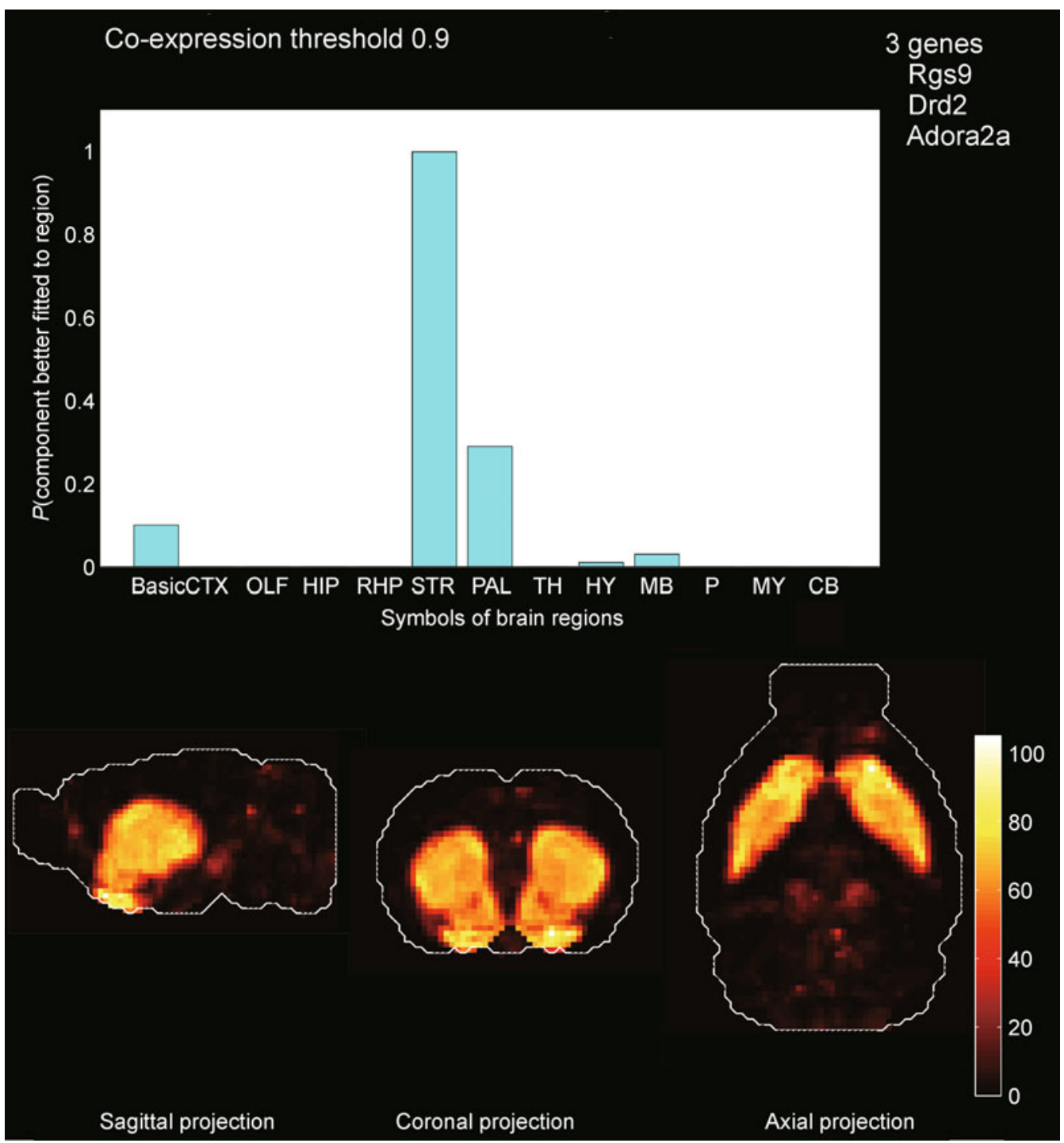

Figure 4. One of the connected components of the co-expression network, at co-expression threshold 0.9. It is better-fitted to the striatum (STR) than more than $99 \%$ of the set of three genes drawn from the coronal Allen Atlas of the adult mouse brain. The symbols for other brain regions read as follows: Basic $=$ 'basic cell groups', CTX = cortex, OLF = olfactory areas, HIP $=$ hippocampal region, $\mathrm{RHP}=$ retrohippocampal region, $\mathrm{PAL}=$ pallidum, $\mathrm{TH}=$ thalamus, $\mathrm{HY}=$ hypothalamus, $\mathrm{MB}=$ midbrain, $\mathrm{P}=$ pons, $\mathrm{MY}=$ medulla, $\mathrm{CB}=$ cerebellum.

\section{Application to a set of addiction-related genes}

The methods reviewed above were applied to a set of 288 genes related to nicotine addiction [11], retrieved from the NicSNP database (http://zork.wustl.edu/nida/Results/ data1.html). The simulation of the cumulative distribution function of co-expression networks of size 288 can be compared to the one of the special set, and plotted together on Figure 7. Since the CDF of the special sets is larger than average at low values of co-expression, the special set is not more co-expressed as a whole than expected by chance. This is confirmed by the statistics of graph properties of networks of 288 genes (Figures 1 and 8). See Ref. [25] for a set of autism-related genes that is more co-expressed in the brain than expected by chance.

However, the graph-based procedure returns special sets when the threshold on co-expression goes from 1 to 0 , which may have exceptional neuroanatomical properties compared to sets of the same size, even if this does not affect the distribution of average and maximal size of connected components. For each of the connected components, the sum of expression energies can also be compared to the partition(s) of the brain given by the ARA, inducing fitting scores in each brain regions (see 


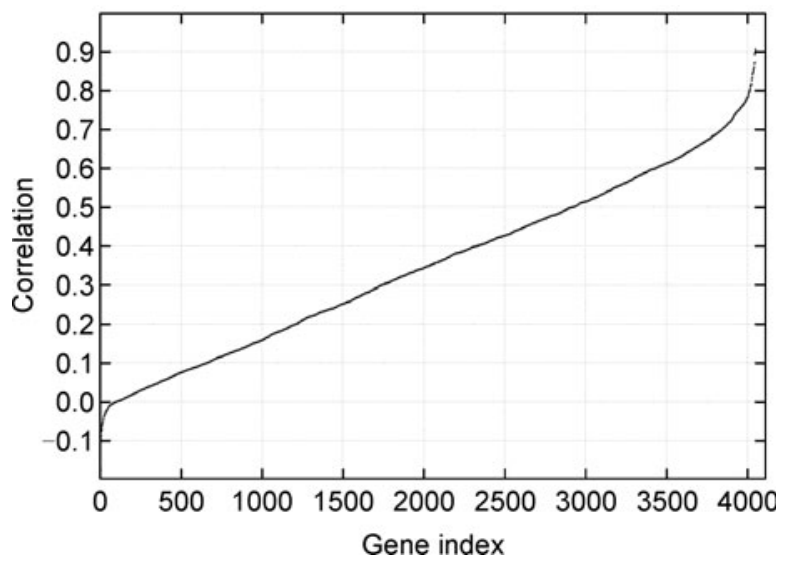

Figure 5. Sorted correlation coefficients between expression energies evaluated from sagittal and coronal sections in the left hemisphere of the mouse brain.

Supplementary Materials S4 for mathematical details). The probability for each connected component of thresholded co-expression networks to have a larger fitting score in a given brain region can be estimated. Imposing a threshold on this probability (99\% for instance) returns sets of genes with exceptional anatomical properties. For the coarsest partition of the left hemisphere, a small set of 3 genes (Rgs9, Drd2, Adora2a) connected at a co-expression of 0.9 , is in the 99th percentile of fitting scores in the striatum (see Figure 4 for a bar diagram of the estimate of $P$-values of fitting scores, and a maximal-intensity projection of the sum of the expression energies of these genes). Even though this set of genes is not exceptional in terms of its size at this value of the co-expression threshold, it has exceptional anatomical properties.

\section{CONCLUSION AND OUTLOOK}

The restriction of the first release of the BrainGeneExpressionAnalysis toolbox to the coronal atlas of the adult mouse brain corresponds to a restriction to genes for which brain-wide data are available. However, the sagittal atlas of the adult mouse brain contains more than 20000 genes, which are included in the NeuroBlast and AGEA tools $[13,14]$. The second release of BGEA will include these genes and restrict the Allen Reference Atlas to voxels where all the genes have ISH data (these voxels correspond to the left hemisphere of the brain). It would also be interesting to estimate the variability of the results under changes of probabilistic universe $C^{\text {Atlas }}$ (by substuting the sagittal atlas to the coronal atlas, and by choosing different image series to construct the data matrix).

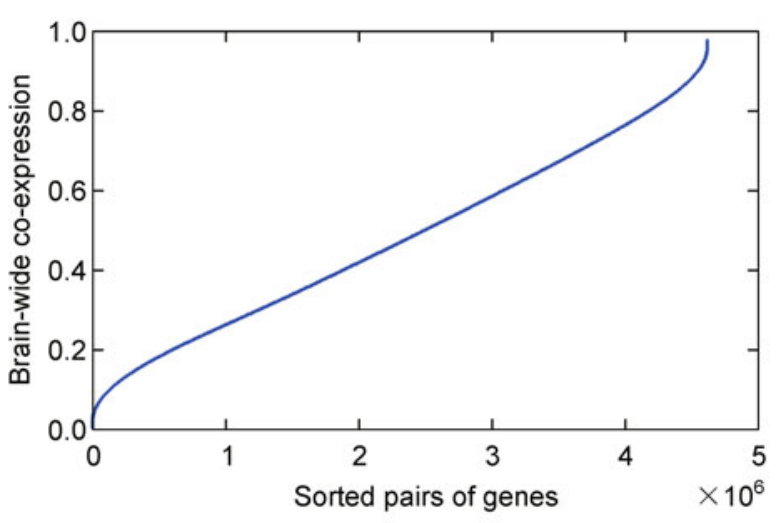

A

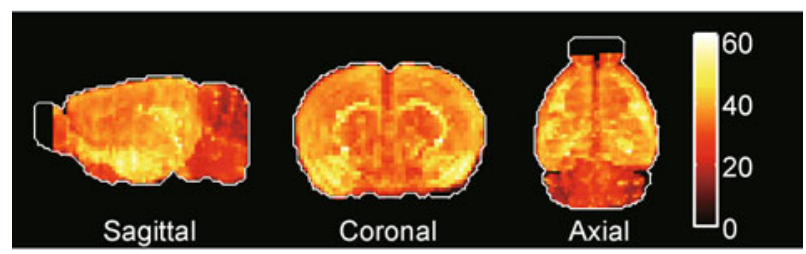

B

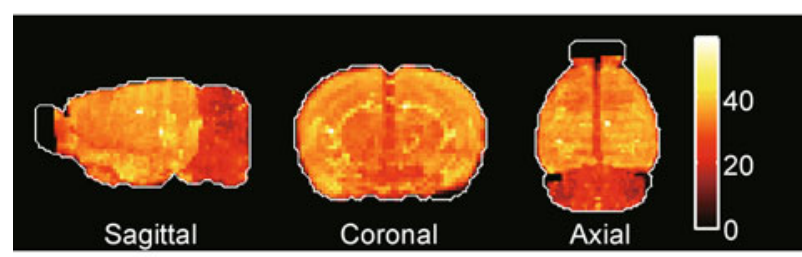

C

Figure 6. (A) Sorted elements of the upper-diagonal part of the co-expression matrix of the coronal atlas, $C^{\text {Atlas }}$. (B) Maximal-intensity projection of the expression energy of Atp6v0c. (C) Maximal-intensity projection of the expression energy of Atp2a2. The pair of genes (Atp6v0c, Atp2a2) has the highest co-expression in the coronal atlas, 0.9781 .

Furthermore, the development of large-scale neuroscience is making comparable atlases available to the research community for other species (see Ref. [26] for the Allen Atlas of the human brain, and Refs. [27,28] for ZEBrA, the Zebra Finch Expression Brain Atlas), and the development of computational resources for the analysis of large data sets can be adapted from the Allen Atlas of the adult mouse brain to other atlases, allowing insights into evolution and into the validity of animal models [29].

Moreover, the size of voxels in the Allen Brain Atlas is large in scale of brain cells, and each voxel may contain cells of different types. Several studies [30-36] have obtained cell-type-specific transcriptional profiles using microarray experiments. Comparison between ISH and microarray data is an ongoing challenge [37], and steps 


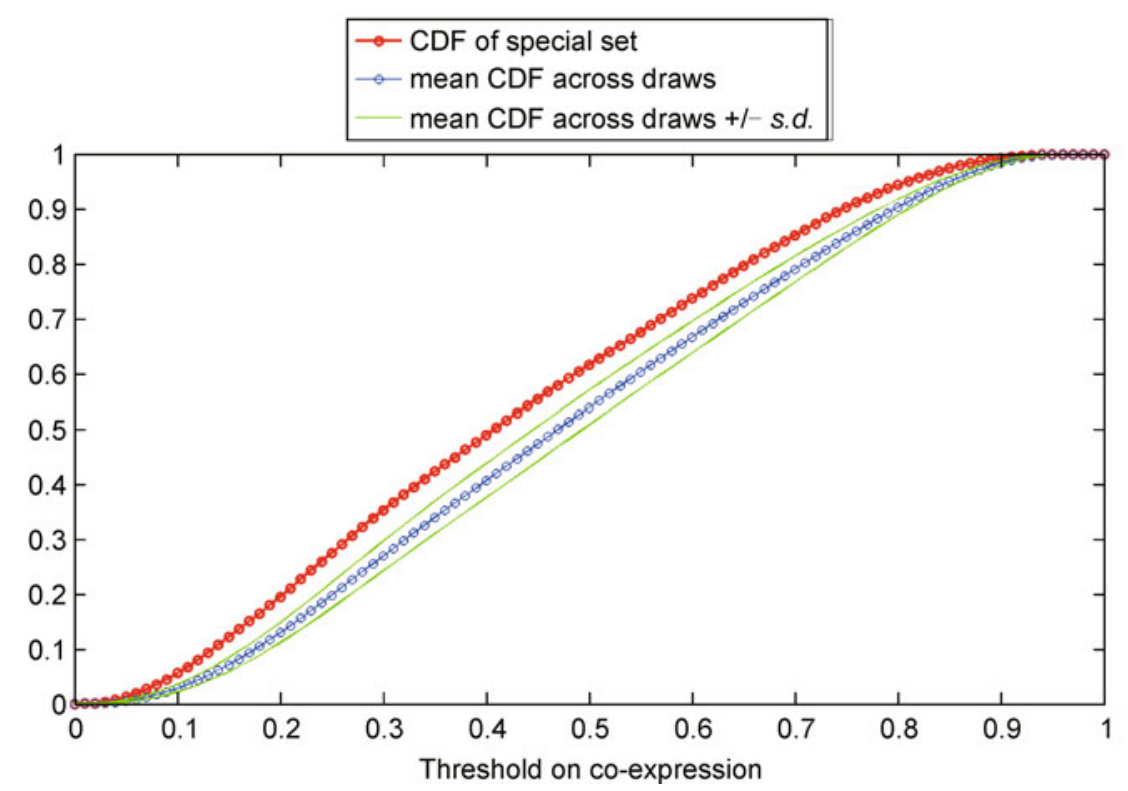

Figure 7. Cumulative distribution function of the upper-diagonal entries of the co-expression matrix of 288 genes (the special co-expression network $C^{\text {set }}$ of the flowchart of Figure 2) from the NicSNP database, for which mouse orthologs are found in the Allen Atlas of the adult mouse brain. As the red curve (or $\mathrm{CDF}^{\mathrm{set}}$ ) sits above the simulated average of the simulated mean of CDFs (or $\langle C D F\rangle$ ) of co-expression networks of 288 genes, at low values of the threshold, the special set as a whole appears to be less co-expressed than expected by chance.

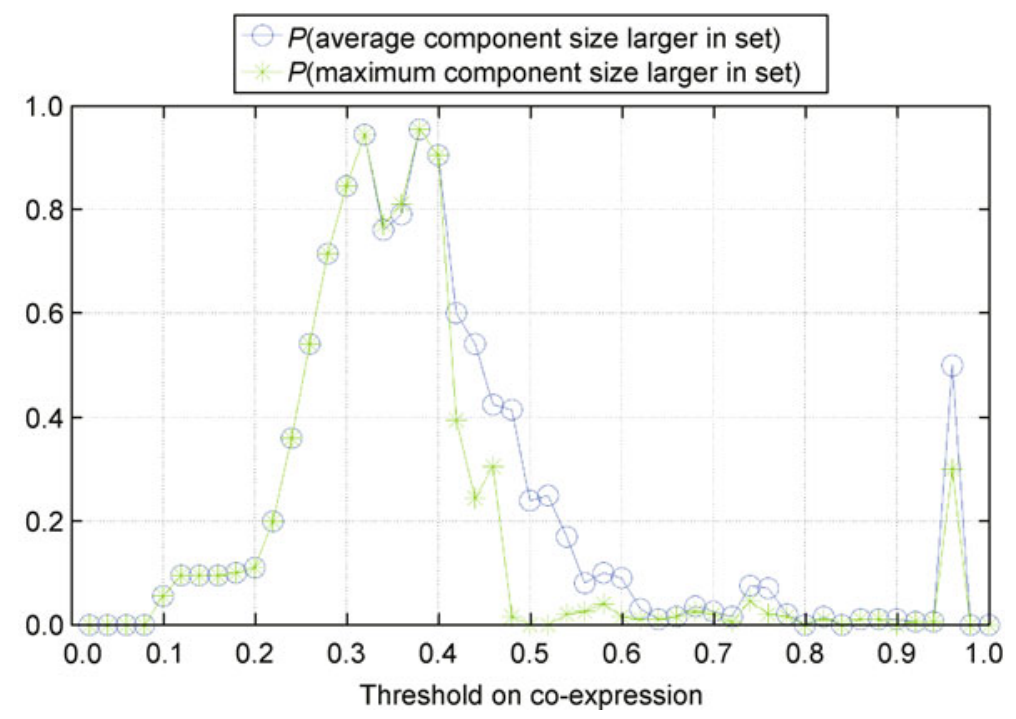

Figure 8. Monte Carlo analysis of the graph underlying the co-expression matrix of 288 genes from the NicSNP database. Estimated probabilities for the average and maximum size of connected components to be larger than in random sets of genes of the same size.

were taken in Ref. [38] to estimate the brain-wide density profiles of cell types by combining the Allen Atlas to the transcriptional profiles of cell types. This sheds light on the cellular origin of co-expression brain-wide coexpression patterns of genes. The corresponding Matlab code will be included in the second release of BGEA.

\section{SUPPLEMENTARY INFORMATION}

Supplementary information includes S1 (Co-expression networks, graphs properties), S2 (Monte Carlo study of gene networks), S3 (Cumulative distribution functions) and S4 (Comparison to classical neuroanatomy), and can 
be found with this article online at DOI 10.1007/s40484013-0011-5.

\section{ACKNOWLEDGEMENTS}

We thank Sharmila Banerjee-Basu, Idan Menashe, Eric C. Larsen, Hemant Bokil and Jason W. Bohland for discussions and collaboration. This research is supported by the NIH-NIDA Grant (1R21DA027644-01, Computational analysis of co-expression networks in the mouse and human brain)

\section{REFERENCES}

1. Bota, M., Dong, H. W. and Swanson, L. W. (2003) From gene networks to brain networks. Nat. Neurosci. 6, 795-799.

2. Lein, E. S., Hawrylycz, M. J., Ao, N., Ayres, M., Bensinger, A., Bernard, A., Boe, A. F., Boguski, M. S., Brockway, K. S., Byrnes, E. J., et al. (2007) Genome-wide atlas of gene expression in the adult mouse brain. Nature 445, 168-176.

3. Sunkin, S. M., Hohmann, J. G. (2007) Insights from spatially mapped gene expression in the mouse brain. Hum. Mol. Genet. 16, 2.

4. Ng, L., Hawrylycz, M. and Haynor, D. (2005) Automated highthroughput registration for localizing 3D mouse brain gene expression using ITK, Insight-Journal.

5. Ng, L., Pathak, S. D., Kuan, C., Lau, C., Dong, H., et al. (2007) Neuroinformatics for genome-wide 3D gene expression mapping in the mouse brain. IEEE/ACM Trans. Comput. Biol. Bioinform. Jul-Sep 4 (3), 382-393.

6. Jones, A. R., Overly, C. C. and Sunkin, S. M. (2009) The Allen Brain Atlas: 5 years and beyond. Nat. Rev. Neurosci. 10, 821-828.

7. Hawrylycz, M., Ng, L., Page, D., Morris, J., Lau, C., Faber, S., Faber, V., Sunkin, S., Menon, V., Lein, E., et al. (2011) Multi-scale correlation structure of gene expression in the brain. Neural Netw. 24, 933-942.

8. Computational analysis of user-defined sets of genes from the Allen Atlas of mouse and human brain can be conducted online at addiction. brainarchitecture.org

9. Grange, P., Bohland, J. W., Hawrylycz, M. and Mitra, P. P. (2012) Brain Gene Expression Analysis: a MATLAB toolbox for the analysis of brain-wide gene-expression data, arXiv:1211.6177 [q-bio.QM].

10. Li, C. Y., Mao, X. and Wei, L. (2008) Genes and (common) pathways underlying drug addiction. PLOS Comput. Biol. 4, e2.

11. Saccone, S. F., Saccone, N. L., Swan, G. E., Madden, P. A. F., Goate, A. M., Rice, J. P. and Bierut, L. J. (2008) Systematic biological prioritization after a genome-wide association study: an application to nicotine dependence. Bioinformatics 24, 1805-1811.

12. Altschul, S. F., Gish, W., Miller, W., Myers, E. W. and Lipman, D. J. (1990) Basic local alignment search tool. J. Mol. Biol. 215, 403-410.

13. Ng, L., Lau, C., Young, R., Pathak, S., Kuan, L., Sodt, A., Sutram, M., Lee, C. K., Dang, C. and Hawrylycz, M. (2007) NeuroBlast: a 3D spatial homology search tool for gene expression. BMC Neurosci. 8, 11.

14. Ng, L., Bernard, A., Lau, C., Overly, C. C., Dong, H. W., Kuan, C., Pathak, S., Sunkin, S. M., Dang, C., Bohland, J. W., et al. (2009) An anatomic gene expression atlas of the adult mouse brain. Nat. Neurosci. $12,356-362$.

15. Zhang, B. and Horvath, S. (2005) A general framework for weighted gene co-expression network analysis. Stat. Appl. Genet. Mol. Biol. 4, e17.

16. Olszewski, P. K., Cedernaes, J., Olsson, F., Levine, A. S. and Schiöth, H. B. (2008) Analysis of the network of feeding neuroregulators using the Allen Brain Atlas. Neurosci. Biobehav. Rev. 32, 945-956.

17. Dong, H. W. (2007) The Allen reference atlas: a digital brain atlas of the C57BL/6J male mouse, Wiley.

18. Grange, P. and Mitra, P. P. (2012) Computational neuroanatomy and gene expression: Optimal sets of marker genes for brain regions. IEEE, in CISS 2012, 46th annual conference on Information Science and Systems (Princeton), arXiv:1205.2721 [q-bio.QM].

19. Lau, C., Ng, L., Thompson, C., Pathak, S., Kuan, L., Jones, A. and Hawrylycz, M. (2008) Exploration and visualization of gene expression with neuroanatomy in the adult mouse brain. BMC Bioinformatics 9, 153.

20. Hawrylycz, M., Baldock, R. A., Burger, A., Hashikawa, T., Johnson, G. A., Martone, M., Ng, L., Lau, C., Larson, S. D., Nissanov, J., et al. (2011) Digital atlasing and standardization in the mouse brain. PLOS Comput. Biol. 7, e1001065.

21. The Allen Brain Atlas can be used online at www.brain-map.org/

22. Tarjan, R. E. (1972) Depth first search and linear graph algorithms. SIAM J. Comput. 1, 146-160.

23. Bohland, J. W., Bokil, H., Pathak, S. D., Lee, C. K., Ng, L., Lau, C., Kuan, C., Hawrylycz, M. and Mitra, P. P. (2010) Clustering of spatial gene expression patterns in the mouse brain and comparison with classical neuroanatomy. Methods 50, 105-112.

24. The developmental atlas of the mouse brain is available from http:// developingmouse.brain-map.org/

25. Menashe, I., Grange, P., Larsen, E. C., Banerjee-Basu, S. and Mitra, P. P. (2012) Co-expression profiling of autism genes in the mouse brain. SFN Abstracts, and submitted.

26. Hawrylycz, M. J., Lein, E. S., Guillozet-Bongaarts, A. L., Shen, E. H., Ng, L., Miller, J. A., van de Lagemaat, L. N., Smith, K. A., Ebbert, A., Riley, Z. L., et al. (2012) An anatomically comprehensive atlas of the adult human brain transcriptome. Nature 489, 391-399.

27. Warren, W. C., Clayton, D. F., Ellegren, H., Arnold, A. P., Hillier, L. W., Künstner, A., Searle, S., White, S., Vilella, A. J. and Fairley, S. (2010) The genome of a songbird. Nature 464, 757-762.

28. Data can be retrieved from the ZEBrA database. (Oregon Health and Science University, Portland, OR 97239; http://www.zebrafinchatlas. org).

29. Miller, J. A., Horvath, S. and Geschwind, D. H. (2010) Divergence of human and mouse brain transcriptome highlights Alzheimer disease pathways. Proc. Natl. Acad. Sci. USA 107, 12698-12703.

30. Rossner, M. J., Hirrlinger, J., Wichert, S. P., Boehm, C., Newrzella, D., Hiemisch, H., Eisenhardt, G., Stuenkel, C., von Ahsen, O. and Nave, K. A. (2006) Global transcriptome analysis of genetically identified neurons in the adult cortex. J. Neurosci. 26, 9956-9966.

31. Cahoy, J. D., Emery, B., Kaushal, A., Foo, L. C., Zamanian, J. L., Christopherson, K. S., Xing, Y., Lubischer, J. L., Krieg, P. A., Krupenko, S. A., et al. (2008) A transcriptome database for astrocytes, neurons, and oligodendrocytes: a new resource for understanding brain development and function. J. Neurosci. 28, 264-278.

32. Doyle, J. P., Dougherty, J. D., Heiman, M., Schmidt, E. F., Stevens, T. R., Ma, G., Bupp, S., Shrestha, P., Shah, R. D., Doughty, M. L., et al. (2008) Application of a translational profiling approach for the comparative analysis of CNS cell types. Cell 135, 749-762.

33. Chung, C. Y., Seo, H., Sonntag, K. C., Brooks, A., Lin, L. and Isacson, O. (2005) Cell type-specific gene expression of midbrain dopaminergic neurons reveals molecules involved in their vulnerability and protection. Hum. Mol. Genet. 14, 1709-1725.

34. Arlotta, P., Molyneaux, B. J., Chen, J., Inoue, J., Kominami, R. and 
Macklis, J. D. (2005) Neuronal subtype-specific genes that control corticospinal motor neuron development in vivo. Neuron 45, 207-221.

35. Heiman, M., Schaefer, A., Gong, S., Peterson, J. D., Day, M., Ramsey, K. E., Suárez-Fariñas M., Schwarz, C., Stephan, D. A., Surmeier, D. J., et al. (2008) A translational profiling approach for the molecular characterization of CNS cell types. Cell 135, 738-748.

36. Sugino, K., Hempel, C. M., Miller, M. N., Hattox, A. M., Shapiro, P., Wu, C., Huang, Z. J. and Nelson, S. B. (2006) Molecular taxonomy of major neuronal classes in the adult mouse forebrain. Nat. Neurosci. 9 ,
99-107.

37. Lee, C. K., Sunkin, S. M., Kuan, C., Thompson, C. L., Pathak, S., Ng, L., Lau, C., Fischer, S., Mortrud, M., Slaughterbeck, C., et al. (2008) Quantitative methods for genome-scale analysis of in situ hybridization and correlation with microarray data. Genome Biol. 9, R23.

38. Grange, P., Bohland, J. W., Bokil, H., Nelson, S., Okaty, B., Sugino, K., $\mathrm{Ng}$, L., Hawrylycz, M. and Mitra, P. P. (2011) A cell-type based model explaining co-expression patterns of genes in the brain, arXiv:1111.6217 [q-bio.QM]. 